Apidologie, 1981, $12(1), 41-55$.

\title{
DER EINFLUSS DER INZUCHT AUF DIE FITNESS DER DROHNEN VON APIS MELLIFERA CARNICA
}

\section{Influence de la consanguinité sur la valeur sélective des mâles d'Apis mellifera carnica}

ROBIN F. A. MORITZ

Institut für Bienenkunde, Im Rothkopf 5, 637 Oberursel (B.R.D.)

\author{
SUMMARY \\ INFLUENCE OF INBREEDING ON THE FITNESS OF DRONES
}

Drones of inbred queens (Apis mellifera carnica) show a significant inbreeding depression. Viability of drones in an incubator and number of spermatozoa are reduced by an increasing inbreeding coefficient. Flightduration experiments show that the age of drones taking their first flight rises by inbred motherqueens. Flightfrequency and dayly flightduration decrease according to the inbreeding coefficient of the dam. A modell with maternal effects seems to be suitable to explain an inbreeding depression on drones.

\section{ZUSAMMENFASSUNG}

Drohnen ingezüchteter Königinnen (Apis mellifera carnica) zeigen mit wachsendem Inzuchtkoeffizienten eine deutliche Inzuchtdepression. Das Überleben im Brutschrank sowie die Anzahl der Spermien nehmen mit steigendem Inzuchtgrad ab. Flugdauerbeobachtungen zeigen, dass das Alter der Drohnen beim ersten Ausflug durch Inzucht der Mutterkönigin erhöht wird. Flugfrequenz und Gesamtflugleistung pro Tag reduzieren sich bei Drohnen ingezüchteter Königinnen. Ein Modell mit maternalen Effekten ist geeignet, die beobachtete Inzuchtdepression bei Drohnen zu erklären.

\section{EINLEITUNG}

Aus der angewandten Tier- und Pflanzenzucht ist bekannt, dass Inzucht zu einer Reduktion der Fitness führt. Unter Fitness ist hier der " reproduktive Erfolg " (die Wahrscheinlichkeit dafür, dass ein Organismus seine Gene an die Nachkommenpopulation weitergibt) zu verstehen. Man spricht auch vom adaptiven 
Wert, der durch Inzucht vermindert wird und bezeichnet diesen Effekt als Inzuchtdepression.

Bei der Honigbiene sind in einer Reihe von Arbeiten Inzuchtdepressionen nachgewiesen worden. PLASs (1953) konnte bei Inzuchtvölkern eine eindeutige Minderung der Fitness beobachten. Die Bruttätigkeit, der Bautrieb und das Verteidigungsverhalten der ingezüchteten Bienen waren reduziert. RoBERTs (1961) fand morphologische Unterschiede zwischen Inzucht- und Hybridbienen und deutet ein grösseres Körpervolumen als Heterozygotenvorteil. Physiologische Aspekte der Inzuchtdepression wurden von BRÜCKNER (1975, 1976, 1978, 1980), von KEPENंA (1978) und von KhISCHA (1978) untersucht. Es konnte in diesen Arbeiten eine deutliche Benachteiligung der Inzuchtlinien im Temperaturregulationsvermögen, im Rekrutierungsverhalten sowie in den Überlebensraten festgestellt werden. OTT und BRÜCKNER (1980) konnten einen Inzuchteffekt auf das Lernverhalten von Arbeitsbienen nachweisen.

Inzuchtdepression wird in der klassischen quantitativen Genetik durch eine Abnahme des Anteils der heterozygoten Loci im diploiden Organismus gedeutet. PIRCHNER (1979) gibt hierzu folgendes Modell :

\begin{tabular}{lcccc} 
genotypischer Wert & $-\mathrm{a}$ & 0 & $\mathrm{~d}$ & $+\mathrm{a}$ \\
\cline { 2 - 6 } Genotyp & + & 1 & + \\
Genfrequenz & $\mathrm{A}_{1} \mathrm{~A}_{1}$ & & $\mathrm{~A}_{1} \mathrm{~A}_{2}$ & $\mathrm{~A}_{2} \mathrm{~A}_{2}$ \\
$\mathrm{q}^{2}$ & & $2 \mathrm{pq}$ & $\mathrm{p}^{2}$
\end{tabular}

Für den genotypischen Wert " $m$ " der Population ergibt sich :

$$
\begin{gathered}
\mathrm{m}=\mathrm{p}^{2} \mathrm{a}+2 \mathrm{pqd}+\mathrm{q}^{2}(-\mathrm{a}) \\
=\mathrm{a}\left(\mathrm{p}^{2}-\mathrm{q}^{2}\right)+2 \mathrm{pqd} \\
\text { mit } \mathrm{p}+\mathrm{q}=1 \text { folgt }
\end{gathered}
$$

$$
\mathrm{m}=\mathrm{a}(\mathrm{p}-\mathrm{q})+2 \mathrm{pqd}
$$

Durch Inzucht wird die Anzahl der heterozygoten Loci vermindert. Der Inzuchtkoefficient " $F$ " gibt die Wahrscheinlichkeit an, mit der zwei Gene an einem Locus durch Inzucht homozygot geworden sind. Im Falle von Inzucht ändert sich die Gleichung 1.2 in

$$
\text { 1.3. } \quad \mathrm{m}_{\mathrm{F}}=\mathrm{a}(\mathrm{p}-\mathrm{q})+2 \mathrm{pqd}(1-\mathrm{F})
$$

Der genotypische Wert einer ingezüchteten Population $\left(\mathrm{m}_{\mathrm{F}}\right)$ wird gegenüber einer nichtingezüchteten um $2 \mathrm{pqdF}$ vermindert sein.

Die Schwerpunkte der bisherigen Arbeiten über Inzucht bei Apis mel. lagen auf der Untersuchung der diploiden Arbeitsbienen, bei denen eine Inzuchtdepression nach Gleichung $1.3 \mathrm{zu}$ erwarten ist. Bei haploiden Drohnen ist eine Erhöhung des Homozygotiegrades nicht möglich und es sollte nach diesem Modell kein Inzuchteffekt nachweisbar sein. Man erhält :

1.4.

$$
\mathrm{m}_{\delta}=\mathrm{a}(\mathrm{p}-\mathrm{q}) \text { mit } \mathrm{m}_{\delta^{*}}=\text { genotypischer Wert } \operatorname{der} \delta \sigma^{\circ}
$$


$\mathrm{m} \sigma^{\top}$ ist dabei unabhängig vom Inzuchtkoeffizienten der betreffenden Königin, der nicht in die Gleichung 1.4 eingeht. Arbeiten von KERR (1969) und BRÜCKNER (1973) scheinen diese These zu bestätigen. Es konnten in diesen Arbeiten keine Hinweise auf eine Inzuchtdepression in der Überlebensrate von Drohnen gefunden werden. Die Variabilität in der Flügelsymmetrie ist nach Inzucht bei den Drohnen nicht vermindert.

Diese Ergebnisse stehen im Gegensatz zu den Daten, die Helle (1965) bei Tetranychus urticae, einer arrhenotoken Milbe, und MoRITZ (1978) bei der KapHonigbiene (Apis mellifera capensis) gefunden haben. HELLE konnte zeigen, dass die Schlüpffähigkeit von Larven aus haploiden Eiern durch Inzucht vermindert wird. Eine Inzuchtdepression manifestiert sich dort sogar noch stärker als in diploiden Eiern. Bei Drohnen von parthenogenetisch erzeugten Inzuchtköniginnen von Apis mellifera capensis konnte bei verschiedenen physiologischen Parametern eine Inzuchtdepression nachgewiesen werden. KHISCHA (1978) fand bei Drohnen der Karpatenbiene ebenfalls Inzuchteffekte im Überleben. Diese Phänomene sind nicht mit der klassischen Inzuchttheorie zu deuten. HeLLE vermutete, dass maternale Effekte eine wesentliche Rolle spielen.

In der vorliegenden Arbeit wird anhand verschiedener Parameter, die die Fitness beschreiben, untersucht, inwieweit sich Inzuchteffekte bei Drohnen ingezüchteter Königinnen manifestieren. Zur Bestimmung der Fitness wird das Überleben unter experimentellen Bedingungen, die Spermienanzahl (als wesentlicher Faktor der Fortpflanzungsfähigkeit) sowie die Flugleistung getestet. Da sich die Drohnen im Flug mit der Königin paaren, ist die Leistungsfähigkeit im Fliegen ein starkes Mass für die Fortpflanzungswahrscheinlichkeit.

\section{MATERIAL UND METHODEN}

\section{INZUCHTLINIEN}

Als Ausgangslinien dienten Stämme von Apis mellifera carnica, deren Abstammung über mehrere Generationen bekannt war, so dass man von definiertem genetischen Material ausgehen konnte. Es wurde zu Beginn der Zucht von Linienkreuzungen ausgegangen, um am Anfang einer jeden Inzuchtlinie einen Inzuchtkoeffizienten $\mathrm{F}=0 \mathrm{zu}$ erzeugen. Die Ausgangslinien waren nicht miteinander verwandt. Mit Hilfe der künstlichen Besamung wurden sechs Inzuchtlinien mit je fünf Inzuchtstufen gezüchtet $(F=0 ; 0,25$; $0,34 ; 0,47 ; 0,5)$. Bei der Haltung der wenig vitalen Inzuchtköniginnen wurde ein " Huckepack "-verfahren angewandt. Die Königinnen wurden in nichtingezüchteten Pflegevölkern gehalten, sodass Verluste durch mangelnde Vitalität der Arbeitsbienen vermieden wurden. Dennoch traten besonders während der Überwinterung häufig Königinnenverluste bis zu $80 \%$ bei den Inzuchtlinien auf.

Die Drohnen, die zu den Experimenten benötigt wurden, befanden sich ebenfalls in nichtingezüchteten Ablegern, um eine ausreichende Pflege zu gewährleisten. In diesen "Drohnenvölkern » befanden sich infolge zweimaliger $\mathrm{CO}_{2}$-Narkose drohnenbrütige Königinnen, sodass man unabhängig von Witterungsund Trachteinflüssen während der gesamten Saison ausreichendes Drohnenmaterial zur Verfügung hatte. Es wurde in den einzelnen Ablegern darauf geachtet, möglichst konstante Volkstärken und Futterverhältnisse zu erhalten, um gleiche Umweltbedingungen bei der Aufzucht der Drohnen zu realisieren. 


\section{METHODEN}

\section{1. Überleben}

Jeweils 50 frisch geschlüpfte Drohnen wurden in ein Holzkästchen (Innenmasse : 6,5 $\times 6,5 \times 4,5 \mathrm{~cm}$ ) gegeben, mit Honig gefüttert und im Brutschrank bei $35^{\circ} \mathrm{C}$ und $40 \%$ relativer Feuchte gehalten. Den Drohnen wurden bewusst keine Pflegebienen zugegeben, um den Leistungsdruck zu verschärfen und auf diese Weise deutlichere Ergebnisse zu bekommen. Im Volk werden frisch geschlüpfte Drohnen in der Regel von Arbeitsbienen gefüttert, während sie in dieser Versuchsanordnung zu selbständiger Futteraufnahme gezwungen waren. Nach $24 \mathrm{~h}$ wurden die toten Drohnen gezählt und das Überleben nach $n / n_{0}$ bestimmt ( $n=$ Anzahl der lebenden $\delta^{\top} \delta^{\star}$ nach $24 \mathrm{~h} ; \mathrm{n}_{0}=$ Anzahl der $\delta^{\top} \delta^{\star}$ bei Versuchsbeginn).

\subsection{Spermienanzahl}

Zur Bestimmung der Anzahl der Spermien pro Drohn wurde die Methode nach EFFINowicz (1978) angewandt :

Begattungsfähige Drohnen, die mindestens elf Tage alt sind, werden mit $\mathrm{CO}_{2}$-Schnee narkotisiert. Man vermeidet dadurch, dass die Drohnen vorzeitig ihren Endophallus evertieren und die Zählung der Spermien unmöglich wird. Die Vesiculae seminales werden herauspräpariert, indem man die Mucusdrüse durchschneidet und die Hoden entfernt. Die isolierten Vesiculae werden danach in ein Blockschälchen mit physiologischer Kochsalzlösung $(1 \mathrm{ml})$ gegeben. Man legt hieran eine $18 \mathrm{~V}$ Wechselspannung. Dies führt zu einer Kontraktion der Vesiculae seminales. Auf diese Weise wird das gesamte Sperma quantitativ ausgepresst. Damit die Spermien im Mikroskop besser sichtbar werden, verdünnt man je nach Spermienzahl mit Aqua dest. bis man eine * zählbare * Spermienkonzentration erhält. Zudem rollen sich die Spermien unter Aqua dest. Einwirkung zusammen und sind dadurch leichter unter dem Mikroskop' erkennbar. Ausgezählt wird in einer Fuchs-Rosenthal-Zählkammer im Phasenkontrastmikroskop bei 200 facher Vergrösserung.

\subsection{Flugleistung}

Die Leistungsfähigkeit im Fliegen wurde anhand der Dauer der Flüge von einzelnen Drohnen bestimmt. Die Drohnen mussten individuell identifizierbar sein, um die exakten Abflug- und Ankunftzeiten feststellen zu können. Hierzu wurden sie mit numerierten Plättchen markiert, wie man sie auch zur Königinnenkennzeichnung benutzt. Die Hauptschwierigkeit bei der Erfassung der einzelnen Flugdaten liegt in der Protokollierung der An- und Abflüge. Da die Drohnen zur Zeit ihrer Hauptaktivität in sehr grosser Zahl ein- und ausfliegen, erwies es sich als notwendig, einen Vorbau vor das Flugloch der Beute zu setzen, der die Beobachtung der einzelnen Drohnen erleichtert (Abb. 1). Es handelt sich um eine Abwandlung des von Drescher (1968) und MaUtz (1970) entwickelten Typs, der die Auftrennung der an- und abfliegenden Bienen erzwingt. Am Flugloch befindet sich ein Drohnenabsperrgitter, das nur während der Beobachtungsperioden entfernt wird. Nichtprotokollierte Drohnenflüge sind dadurch ausgeschlossen.

\section{ERGEBNISSE}

\section{1. $\ddot{U B E R L E B E N}$}

Insgesamt 4400 Drohnen aus drei verschiedenen Inzuchtlinien mit den Inzuchtkoeffizienten $\mathrm{F}=0 ; 0,34 ; 0,47 ; 0,5$ wurden getestet. Abbildung 2 zeigt die Abhängigkeit des Überlebens vom Inzuchtgrad der drohnenproduzierenden Königin. Eine Regressionsanalyse zeigt, dass das Überleben signifikant mit zunehemendem Inzuchtkoeffizienten abnimmt $\left(\hat{\beta}=-0,58 ; p=0,01\right.$ bei $\left.\mathrm{H}_{0}: \beta=0, t-T e s t\right)$. Ein Inzuchteffekt auf die Fitness der Drohnen kann bei diesem Parameter eindeutig gezeigt werden. 


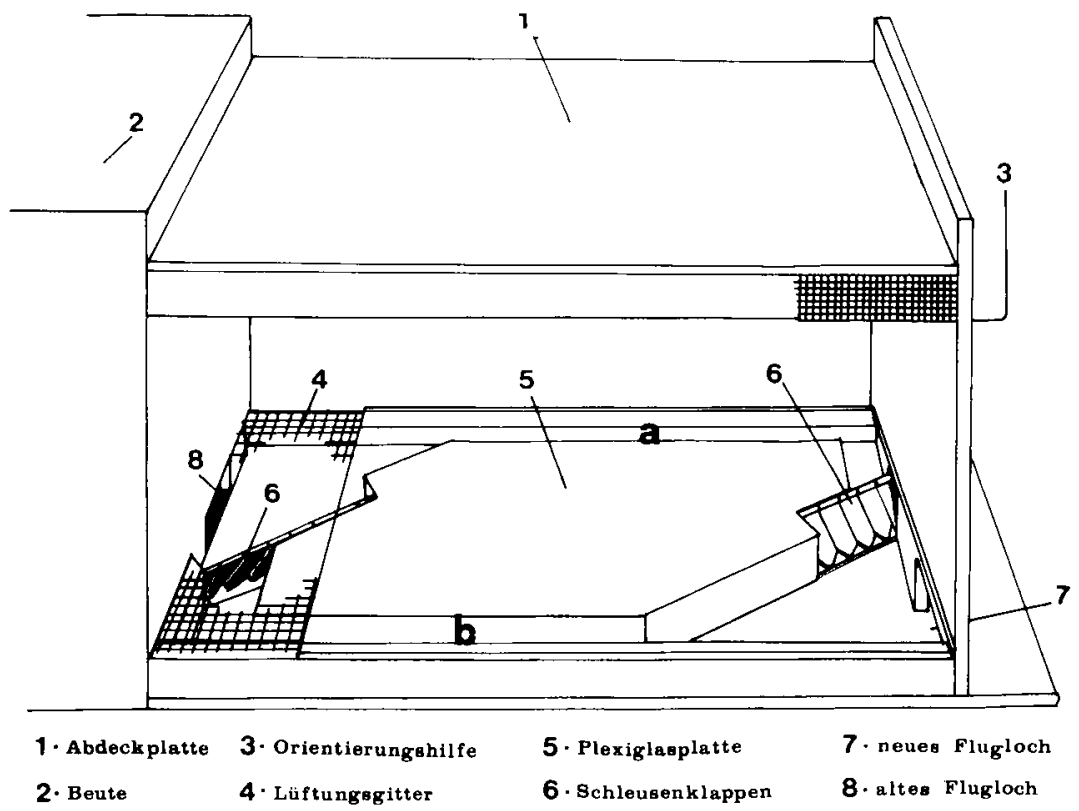

Авв. 1. - Vorbau an der Beute zur Auftrennung der an- und abfliegenden Bienen.

Der Klappenmechanismus (6) bewirkt, dass die ausfliegenden Bienen

die Beute durch " $a$ " verlassen, während die ankommenden durch " $b$ " hindurchlaufen.

Eine Holzplatte an der Vorderseite (3)

ist in Form und Farbe der originalen Beutenfront gleich.

Dies erleichtert den Bienen die Orientierung. Eine Abdeckplatte (1)

baut innerhalb des Vorbaus einen Helligkeitsgradienten auf, der zum Flugloch (7) hin zunimmt und ebenfalls

der Orientierungserleichterung dient. Eine Drahtgaze (4) sorgt für ausreichende Lüftungsmöglichkeit.

FIG. 1. - Avant-corps de la ruche destiné à séparer les abeilles qui sortent de celles qui rentrent.

Les clapets (6) font entrer les abeilles par " $a$ " et les font sortir par " $b$ ".

Sur la façade une planche de bois reproduit en forme et en couleurs le fronton d'origine.

Ceci facilite l'orientation des abeilles. Une plaque de couverture mobile (3) forme

à l'intérieur de l'avant-corps un gradient de luminosité qui croît jusqu'au trou de vol (7) et sert également à faciliter l'orientation.

Une gaze métallique (4) assure une aération éventuelle suffisante.

1. Plaque de couverture mobile

2. Ruche

3. Aide pour l'orientation

4. Grille d'aération
5. Plaque de plexiglass

6. Clapets

7. Nouveau trou de vol

8. Ancien trou de vol.

\section{SPERMIENANZAHL}

Bei der Untersuchung der Anzahl der Spermien pro Drohn wurden ebenfalls drei verschiedene Inzuchtlinien mit den Inzuchtstufen $F=0 ; 0,25 ; 0,34 ; 0,5$ herangezogen. Je Inzuchtstufe wurden 50 Drohnen untersucht. Es zeigte sich in der 


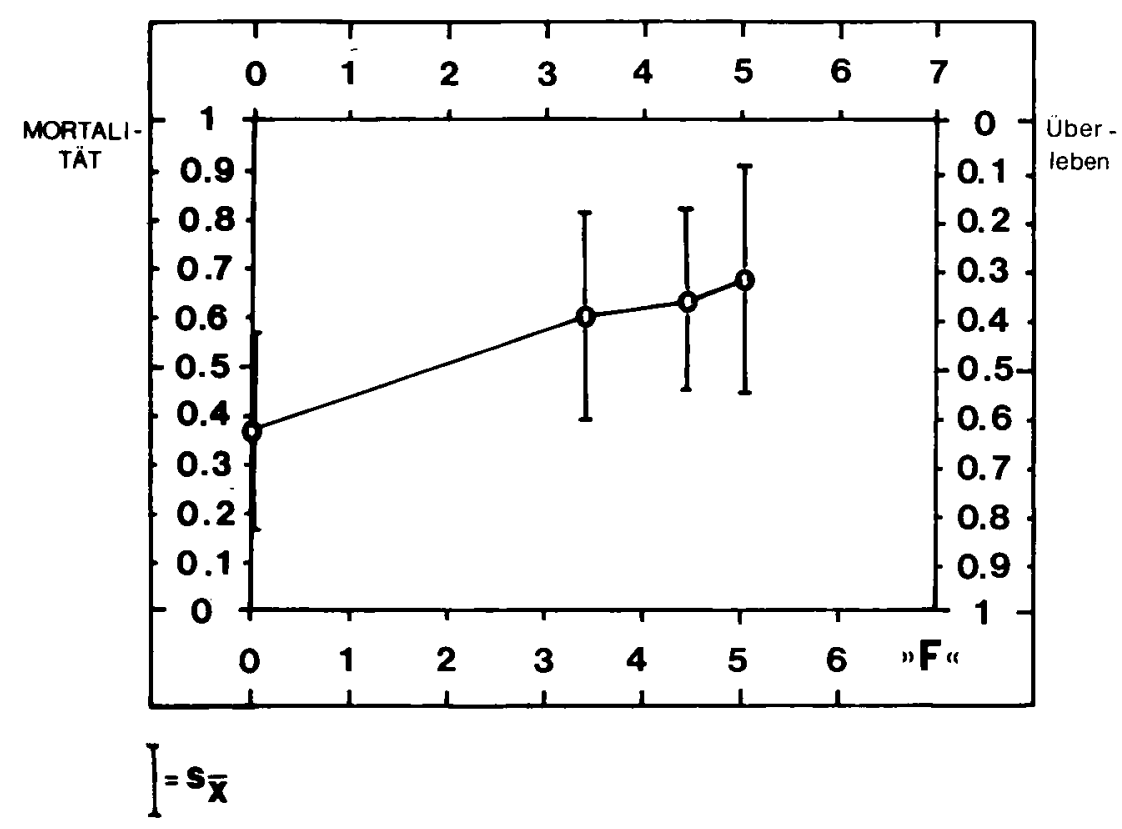

Aв8. 2. - Abhängigkeit des Überlebens von Drohnen vom Inzuchtkoeffizient der Mutterköniginnen.

Die Punkte stellen die Mittelwerte von je 22 Messungen (+ 1100 Drohnen) dar.

FIG. 2. - Relation entre la longévité des mâles et le coefficient de consanguinité de la reine mère. Chaque point représente la moyenne de 22 mesures $(=1100$ mâles).

Regressionsanalyse, dass eine nahezu lineare Abhängigkeit von Spermienzahl zu Inzuchtkoeffizient vorlag (Abb. 3). Der t-Test belegt, dass der Regressionskoeffizient $(\hat{\beta}=-13,1)$ auf dem 0,001 Niveau signifikant von Null verschieden ist.

\section{FLUGLEISTUNGEN}

Die Versuche zur Bestimmung der verschiedenen Flugleistungen wurden in der Zeit vom 28.6.-20.7.1977 sowie vom 7.7.-23.7.1978 in Oberursel durchgeführt. Da warme Temperaturen (temp. max. $=25^{\circ} \mathrm{C}$ ) und sonniges Wetter vorherrschten, waren die Bedingungen zu regem Drohnenflug erfültt.

\subsection{Alter beim ersten Ausflug}

Je Inzuchtstufe $(F=0 ; 0,25 ; 0,34 ; 0,47 ; 0,5)$ wurden 100 Drohnen markiert und individuelle Flugprotokolle angefertigt. Abbildung 4 zeigt die Abhängigkeit des Alters beim ersten Flug von den jeweiligen Inzuchtkoeffizienten. Da Witterungsbedingungen sehr starken Einfluss auf den ersten Ausflug wie auf den Drohnenflug generell haben, wurde es notwending die Daten zu standardisieren. Das Alter der nichtingezüchteten 


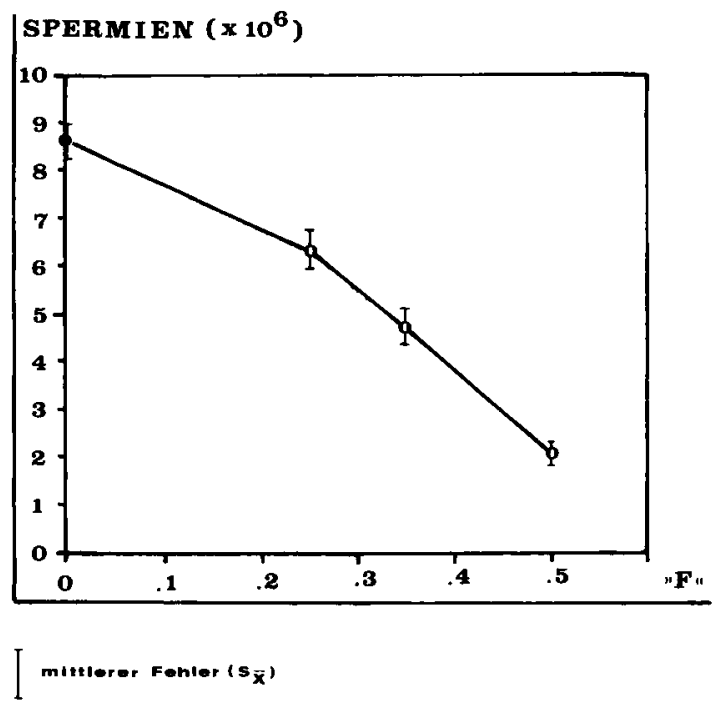

Авв. 3. - Spermienzahl bet Drohnen von ingezüchteten Königinnen in Abhängigkeit des Inzuchtkoeffizienten.

FIG. 3. - Relation entre le nombre de spermatozoïdes chez les mâles provenant de reines consanguines et le coefficient de consanguinité.

\section{AUSFLUG}

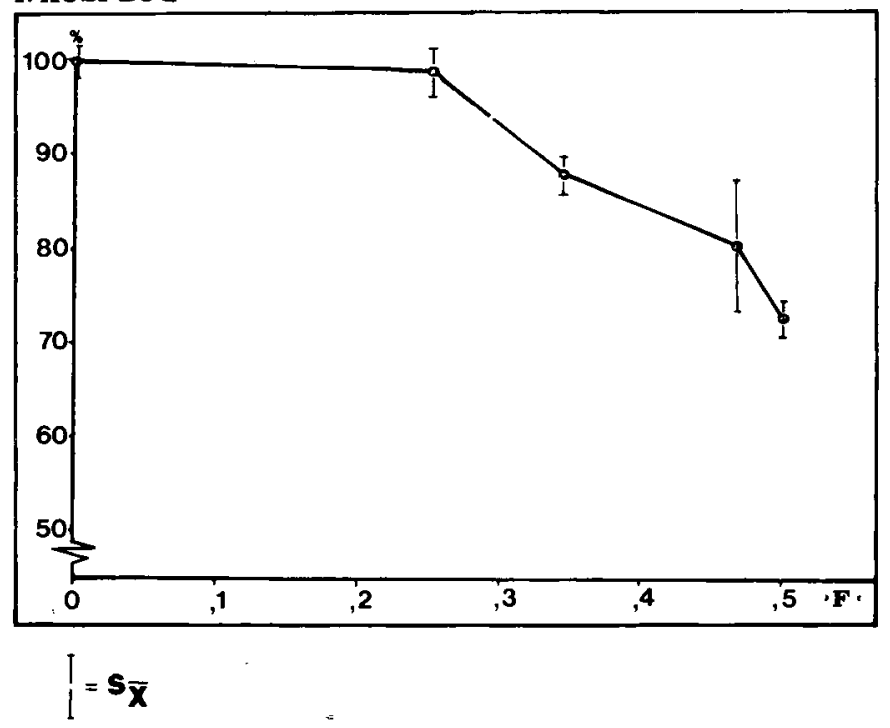

Авв. 4. - Abhängigkeit des Drohnenalters beim ersten Ausflug vom Inzuchtkoeffizient der Mutterkönigin. Die Ordinate gibt die Flugleistung in \% an, wobei das Alter der Kontrolle ( $\mathrm{F}=0$ ) mit $100 \%$ angesetzt wurde.

FIG. 4. - Relation entre l'âge des mâles à leur $1^{\text {er }}$ vol et le coefficient de consanguinité de la reine. Le facteur âge des mâles au $1^{\text {er }}$ vol est donné en ordonnée en $\% .100 \%$ est attribué aux témoins $(F=0)$. 
Kontrolldrohnen wurde gleich 1 gesetzt und die prozentuale Abweichung der Inzuchtdrohnen ermittelt. Das durchschnittliche Alter der Kontrollgruppe lag witterungsbedingt mit 11,1 Tagen über den in der Literatur beschriebenen Werten (Drescher 1968, Howell und Usinger 1933, KuRENNoI 1954, Kepena 1963). Bei einem Inzuchtkoeffizienten von 0,5. hat sich das Alter auf 14,25 Tage erhöht, was einer Leistungsverminderung um $27,7 \%$ entspricht. Die Witterungsabhängigkeit des Datenmaterials bedingt, dass keine Normalverteilung zu grunde gelegt werden kann. Eine Rangkorrelationsanalyse nach Spearman ergibt $r_{s}=-0,87$ und Signifikanz bei $\mathrm{p}=0.01$. Bei ihrem ersten Ausflug verhalten sich die Drohnen ähnlich dem Vorspiel der jungen Arbeitsbienen, was auch schon Drescher (1968) und WITHERELl (1970, 1971) beobachten konnten. Sie fliegen innerhalb Sichtweite der Beute mit dem Kopf zum Flugloch umher. Vor dem Ausflug kann intensives Putzverhalten beobachtet werden.

\subsection{Paarungsflugfrequenz}

Je öfter ein Drohn auf den Drohensammelplatz ausfliegt, um so grösser wird seine Chance auf Paarungserfolg sein. Nur Flüge begattungsfähiger Drohnen (älter als 11 Tage), die länger als zehn Minuten dauerten, wurden in die Auswertung aufgenommen, da diese besondere Relevanz für die Fitness haben. Auch hier wurde das Datenmaterial standardisiert, um die unterschiedlichen Witterungseinflüsse auszuschliessen. Abb. 5

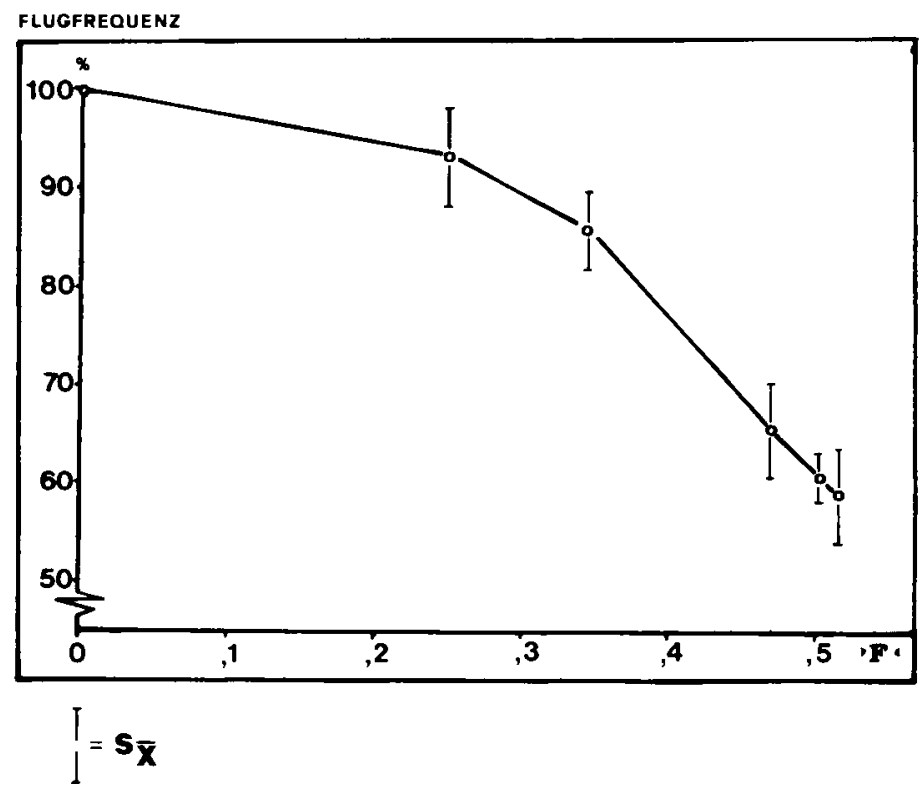

ABB. 5. - Abhängigkeit der Drohnenflugfrequenz vom Inzuchtkoeffizient der Mutterkönigin. Die Flugfrequenz der Kontrolle wurde gleich $100 \%$ gesetzt.

FIG. 5. - Relation entre la fréquence de vol des mâles et le coefficient de consanguinité de la reine. La fréquence de vol des témoins est fixée à $100 \%$. 
zeigt den Zusammenhang zwischen Flugfrequenz und Inzuchtkoeffizient der Königin, die die Drohnen produzierte. Der Median der Kontrollgruppe lag bei 5 Flügen pro Tag $(R=7)$. Bei einem Inzuchtkoeffizient von 0,51 ist die Flugleistung um $41,75 \%$ reduziert. Zur statistischen Auswertung wurde die paramterfreie Rangkorrelationsanalyse nach Spearman angewandt. $r_{s}=-0,98$ ist auf dem 0,01 Niveau signifikant von Null verschieden und bestätigt den negativen Zusammenhang der Graphik zwischen Inzuchtkoeffizient und Flugfrequenz.

\subsection{Gesamtflugleistung pro Tag}

Als stärkstes Mass für die Fitness ist im Rahmen der Flugdauerbeobachtungen die Gesamtflugleistung eines Drohns pro Tag anzusehen. Die Gesamtflugleistung pro Lebensdauer eines Drohn kann im Freiland nur von untergeordneter Bedeutung sein. Drohnen sterben nach Eversion des Endophallus bei der Paarung. Der Aspekt Lebensdauer ist somit im Freiland für eine Bestimmung der Fitness irrelevant, da je nach Ursachen des Todes zugleich grösstmögliche Fitness (erfolgreiche Begattung einer Königin) als auch stark verminderte Fitness (Tod auf dem Paarungsflug) vorliegen kann. Zudem wird sich im Laufe des Experiments die Zahl der markierten Drohnen auch durch Verflug in benachbarte Stände reduzieren. Eine Bestimmung der Lebensdauer an Hand der Zahl der gekennzeichneten Drohnen im Versuchsvolk erscheint nicht sinnvoll. Bei der Bestimmung der täglichen Gesamtflugleistung wurden nur die Drohnen berücksichtigt, die bei Versuchsende wieder ins Volk zurückgekehrt waren. Es wurde nur die Summe der Paarungsflugzeiten gebildet. Orientierungsflüge ( $\leqq 10 \mathrm{~min}$ ), bei denen die Wahrscheinlichkeit einer erfolgreichen Begattung nur gering ist, wurden von der täglichen Gesamtflugleistung abgezogen. Man erhält so ein Mass für die Dauer der Präsenz der Drohnen auf dem Drohnensammelplatz, die die Wahrscheinlichkeit auf eine erfolgreiche Begattung bestimmt. Abb. 6 zeigt, dass mit zunehmendem Inzuchtkoeffizient die Fitness in Form einer reduzierten Flugleistung abnimmt. Die Gesamtflugleistung ist bei $F=0,51$ um 41,1 \% vermindert. Eine Analyse nach Spearman ist mit $r_{s}=-0,99$ signifikant $(p=0,01)$. Wie oben handelt es sich auch hier um standardisiertes Datenmaterial. Die Flugleistung der nichtingezüchteten Kontrolldrohnen wurde gleich 1 gesetzt.

\subsection{Flugdauer pro Flug}

Dies ist neben der Flugfrequenz der zweite Parameter, der die tägliche Gesamtflugdauer bestimmt. Es wurden wiederum nur die Paarungsflüge berücksichtigt. Man kann dem graphischen Verlauf keinen Zusammenhang zwischen Inzuchtkoeffizient und Flugdauer pro Paarungsflug entnehmen (Abb. 7). Die Rangkorrelationsanalyse nach Spearman kann die Nullhypothese, " $F$ " und Flugdauer/Flug seien unabhängig, nicht widerlegen $\left(r_{s}=0,11\right)$. Es zeigt sich, dass die Flugfrequenz als Ursache der reduzierten Gesamtflugleistung bei den Inzuchtdrohnen anzusehen ist. Dies wird durch die Graphik der Abb. 8 verdeutlicht, in der die 


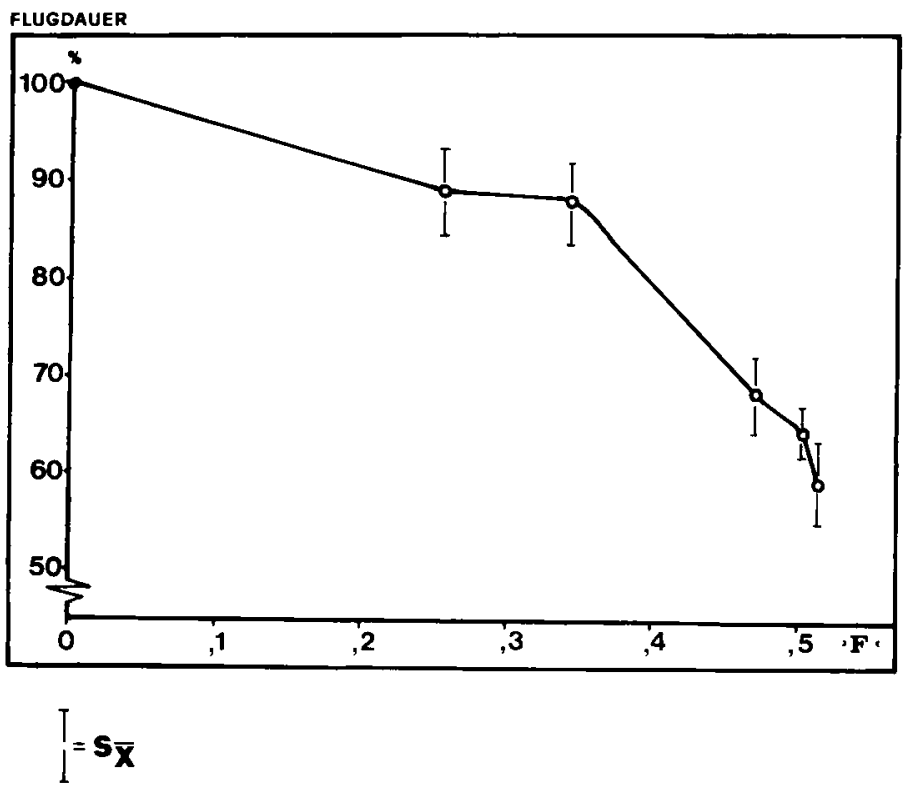

Aвв. 6. - Abhängigkeit der täglichen Paarungsflugdauer von Drohnen ingezüchteter Königinnen vom Inzuchtkoeffizienten.

Die Flugleistung der Kontrolle beträgt $100 \%$.

FIG. 6. - Relation entre la durée journalière des vols nuptiaux des mâles provenant de reines consanguines et le degré de consanguinité.

La durée de vol des témoins atteint $100 \%$.

FLUGDAUER/ FLUG

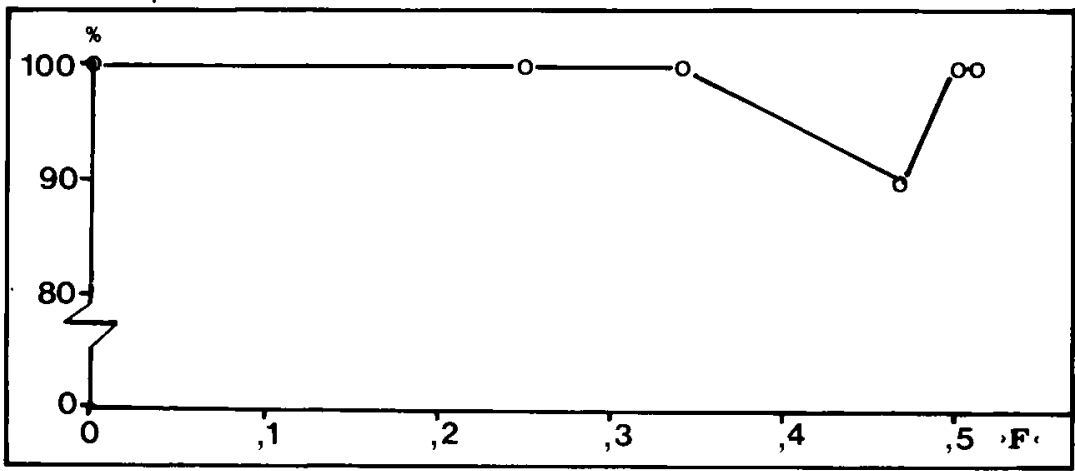

Авв. 7. - Flugdauer pro Paarungsflug von Drohnen ingezüchteter Königinnen in Abhängigkeit des Inzuchtkoeffizienten. Die Flugdauer der nichtingezüchteten Kontrolldrohnen wurde gleich $100 \%$ gesetzt.

FIG. 7. - Durée de chaque vol nuptial de mâles provenant de reines consanguines en relation avec le coefficient de consanguinité.

La durée de vol des mâles témoins non consanguins est fixé à $100 \%$. 


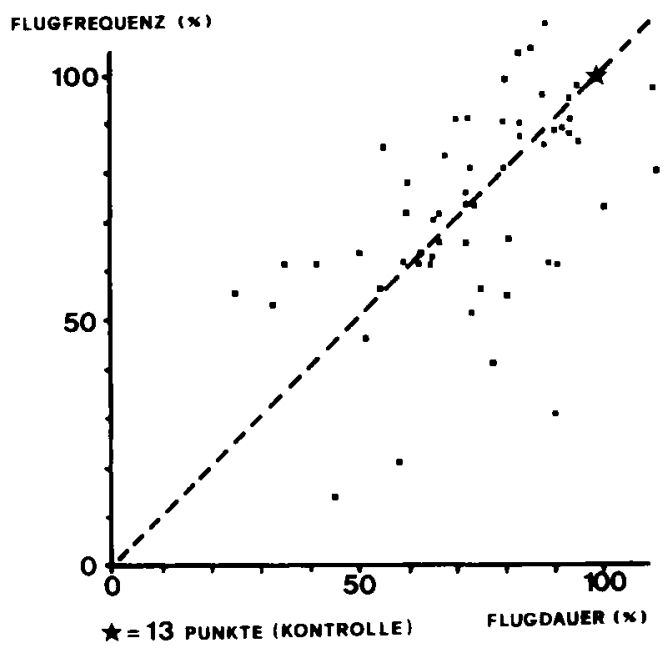

ABB. 8. - Zusammenhang zwischen Flugfrequenz und tägliche Gesamtflugdauer von Drohnen. Die Punkte in der Punktwolke sind die Mittelwerte einer Drohnengruppe (Drohnen einer Inzuchtstufe) an jeweils einem Flugtag.

Fig. 8. - Relation entre la fréquence de vol (en ordonnées) et la durée totale journalière de vol des mâles (en abscisses).

Les points représentent les moyennes d'un groupe de mâles

(mâles d'un degré de consanguinité) pour chaque jour de vol.

Abhängigkeit der Gesamtflugdauer von der Flugtrequenz dargestellt ist. Der spearmansche Rangkorrelationskoeffizient $r_{s}=0,99$ ergibt Signifikanz auf dem 0,01 Niveau.

\section{DISKUSSION}

In allen untersuchten Parametern konnte ein deutlicher Zusammenhang zwischen Fitness der Drohnen und Inzuchtkoeffizient der Mutterkönigin gezeigt werden. Beim Überleben und bei der Anzahl der Spermien sind lineare Abhängigkeiten der Fitness vom Inzuchtgrad nachgewiesen worden, wie dies auch bei Drosophila (DoBZHANSKI 1963) und vielen anderen Organismen der Fall ist. Nimmt man das Datenmaterial von KHISCHA (1978) und setzt es zum Inzuchtkoeffizienten im Bezug, so kann man bei den Arbeitsbienen an den Parametern Flügellänge und Flügelbreite ebenfalls einen relativ linearen Zusammenhang zwischen Merkmal und Inzuchtkoeffizient finden. In den Arbeiten von TANTAWAY und REEVE (1956) werden jedoch auch nichtlineare Zusammenhänge beobachtet. Bei Drosophilapopulationen konnten die Verfasser zeigen, dass bei schwacher Inzucht eine geringe, bei starker aber eine überproportionale 
Leistungsdepression auftrat. KнISCHA (1978) gibt ebenfalls Daten für nichtlineare Beziehungen zwischen Merkmal und Inzuchtkoeffizient. Überleben von diploiden Larven, Königinnengewicht und Ovariolenzahl, sowie Arbeiterinnengewicht und Tergitlänge scheinen einer exponentiellen Regressionskurve zu folgen, die dem Typus $y=a x^{b e}$ zuzuordnen ist. In der vorliegenden Arbeit können mit den Daten, die zur Bestimmung der Flugdauer gewonnen wurden, ebenfalls nichtlineare Abhängigkeiten zwischen Flugleistung und Inzuchtgrad dargestellt werden. Ein solcher Zusammenhang könnte die Ergebnisse von KERR (1969) und BRÜCKNER (1973) erklären, die in ihren Arbeiten keine Hinweise auf Inzuchteffekte finden konnten. Ein zu schwacher Inzuchtgrad der Mutterköniginnen könnte als Ursache für die Nichtausprägung einer Inzuchtdepression bei den Drohnen angesehen werden.

Es zeigt sich, dass die bei Drohnen gefundenen Abhängigkeiten zwischen Inzuchtgrad und Merkmal bei Arbeitsbienen im gleichen Masse auftreten. Eine Inzuchtdepression bei diploiden Arbeitsbienen ist nach dem eingangs dargestellten Modell durchaus erkläbar. Die Inzuchteffekte, die bei den Drohnen gefunden wurden, liegen ausserhalb dieses Modells, da dies nur im diploiden Fall Gültigkeit hat. Ein entsprechendes haploides Modell sieht wie folgt aus :

\begin{tabular}{lccc} 
genotypischer Wert & $-a$ & 0 & $+a$ \\
\cline { 2 - 4 } Genotyp & $A_{1}$ & + \\
Genfrequenz & $q$ & $A_{2}$ \\
& $q$ & $p$
\end{tabular}

Für den genotypischen Wert der Drohnen erhält man, wie bereits in der Einleitung erwähnt

4.1

$$
\begin{aligned}
\mathrm{m} & =\mathrm{pa}-\mathrm{qa} \\
& =(\mathrm{p}-\mathrm{q}) \mathrm{a}
\end{aligned}
$$

Das bedeutet, dass sich der genotypische Wert der Drohnen nur durch Genfrequenzverschiebungen erklären liesse, da a konstant ist. Genfrequenzverschiebungen treten auf durch Mutation, Selektion, Migration und genetische Drift.

Migration kann ausgeschlossen werden, da nach Versuchsbeginn kein genetisches Material von aussen hinzukam oder entfernt wurde. Mutationen sind zu vernachlässigen, da es sich um polygene Merkmale handelt, die untersucht wurden. Die Wahrscheinlichkeit für eine Spontanmutation solcher Merkmale ist äusserst gering. Selektion wurde vermieden. Der genetischen Drift wurde dadurch begegnet, dass mehrere Linien in das Versuchsprogramm aufgenommen wurden. Die genetische Drift ist ein Zufallsprozess, so dass bei sechs getesteten Linien die Wahrscheinlichkeit für eine gleiche Drift in Richtung einer Fitnessreduktion bei Drohnen 0,016 beträgt (1 Locus).

Die Parameter, die nach dem quantitativ genetischen Modell eine Inzuchtdepression deuten können sind demnach im hier beschriebenen Fall von nur untergeordneter Bedeutung. 
Eine Möglichkeit der Interpretation der beobachteten Inzuchtphänomene besteht in einem von HeLLe (1965) vorgeschlagenen Modell, indem maternale Effekte einen wichtigen Faktor darstellen. Man geht davon aus, dass mütterliche Proteine und Enzyme einen Einfluss auf die Fitness der Nachkommen haben. MAUL (1969) konnte zeigen, dass sich die letale Kreuzung Apis mellifera $\times$ Apis cerana nach der Befruchtung bis zum Blastodermstadium entwickelt. MAUL führt dies auf den bis zu diesen Stadium funktionierenden maternalen Enzymapparat zurück. ToBARI und MORIWAKI (1963) beobachteten bei Drosophila ananassae einen maternalen Einfluss auf die Fitness der Nachkommen. Nach FISCHER (1978) ergibt sich folgender Modellansatz :

Es ist bekannt, dass Hybridenzyme bei zunehmender Homozygotie nicht mehr auftreten. Ingezüchtete Königinnen sollten ein reduziertes Enzymspektrum zeigen, das sich auch im Eiprotein dokumentieren sollte. Ein negativer Effekt auf die Fitness der Nachkommen wäre somit wahrscheinlich. Jedoch muss bei diesem Modell gesehen werden, dass gerade bei der Biene Isoenzympolymorphismen in Folge der haplodiploiden Populationsstruktur, vermindert sind (HARTL 1971; SNYDER 1974; BRÜCKNER 1973). Beim Fehlen polymorpher Loci können keine Hybridenzyme auftreten, so dass ein maternaler Inzuchteffekt wie oben beschrieben nicht in Erscheinung treten könnte. Allerdings scheint trotz einer Reduktion des Polymorphismus bei Hymenopteren, dieser nicht vollständig zu fehlen, wie die Arbeiten von Metcalf (1975), Halliday (1978), Lester und Selander (1978) und PAMILO et al. (1978) gezeigt haben. Eine Reduktion des maternalen Enzymspektrums durch Inzucht wäre somit zu erwarten und ein plasmatisch bedingter Inzuchteffekt auf die Nachkommen möglich.

Eingegangen im Oktober 1980. Reçu pour publication en octobre 1980.

\author{
RÉSUMÉ \\ INFLUENCE DE LA CONSANGUINITÉ SUR LA VALEUR SELECTIVE \\ DES MALES D'APIS MELLIFERA CARNICA
}

La dépression consanguine est expliquée dans la théorie classique (PIRCHNER, 1979) par l'augmentation de la part des loci homozygotes dans l'organisme diploïde. Une augmentation du degré d'homozygotie n'est toutefois pas possible chez les individus haploïdes. Les mâles haploïdes de reines consanguines ne devraient donc pas présenter une dépression consanguine.

On a testé des mâles de reines consanguines provenant de 6 lignées consanguines avec 5 degrés de consanguinité $(\mathrm{F}=0 ; 0,25 ; 0,34 ; 0,47 ; 0,5)$. L'étude de la durée de vie en étuve de mâles récemment éclos a montré l'existence d'une relation nette avec le coefficient de consanguinité de la reine. Lorsque la consanguinité augmente, la durée de vie diminue ( $\hat{\beta}=-0,58$ ) (Fig. 2).

Il existe une régression linéaire négative entre le nombre de spermatozoīdes par mâle et le coefficient de consanguinité de la reine $(\hat{\beta}=-13,1)$ (Fig. 3 ). 
Les observations sur la durée de vol en conditions naturelles avec des mâles marqués ont montré que la capacité de vol diminuait lorsque le degré de consanguinité augmentait.

L'âge des mâles à leur premier vol augmente en même temps que le coefficient de consanguinité de la reine diminue. On obtient un coefficient de corrélation de rang (SPEARMAN) entre la capacité de vol et le degré de consanguinité de $r_{8}=-0,87$ (Fig. 4).

La fréquence des vols nuptiaux par jour diminue nettement chez les mâles provenant de reines consanguines. On obtient une corrélation entre le degré de consanguinité et la réduction de la fréquence de vol : $r_{s}=-0,98$ (Fig. 5).

La durée journalière du vol nuptial diminue également lorsque croît le coefficient de consanguinité. On obtient une corrélation négative de $r_{s}=-0,99$ (Fig. 6.). Cela prouve que c'est la fréquence des vols nuptiaux et non la durée de vol de chacun d'eux qui provoque la réduction de la capacité globale du vol par jour (Fig. 7, Fig. 8).

En contradiction avec le modèle cité plus haut on a pu décrire une relation entre la valeur sélective des mâles et le coefficient de consanguinité de la reine. Un modèle, dans lequel les effets maternels ont une forte influence sur la descendance, expliquerait dans ce cas les effets de consanguinité trouvés chez les mâles.

\section{LITERATURVERZEICHNIS}

BRÜCKNER D., 1973. - Homöostase bei Honigbienen. Die Naturwissenschaften, 60, 433-434.

BRỨcKNER D., 1975. - Die Abhängigkeit der Temperaturregulierung von der genetischen Variabilität der Honigbiene (Apis mel.). Apidologie 6, 361-380.

BRÜCKNER D., 1976. - Nachweis von Heterosis für das Merkmal Temperaturregulierung bei der Honigbiene (Apis mel.) Apidologie 7, 243-248.

BRÜCKNER D., 1978. - Why are there inbreeding effects in haplo-diploid systems? Evolution 32, 456-458.

BRÜCKNER D., 1980. - Hoarding behaviour and life span of inbred, non inbred and hydrid honeybees (Apis mel.) J. apic. Res. 19 (1).

Dobzhansky Th., Spassky B., 1963. - Genetics of Populations XXXIV. Adaptive norm, genetic load and genetic elite in Drosophila pseudobscura. Gen. 48, 1467-1485.

Drescher W., 1968. - Die Flugaktivität von Drohnen der Rassen Apis mel. carnica und Apis mel. ligustica. Z. f. Bienenforschung 9, 390-409.

EFFiNowicz G., 1978. - Einfluss der Temperatur auf die sexuelle Reifung der Drohnen. Referat auf der Tagung der AG Deutsche Bieneninstitute in Münster 1978.

Fischer H. E., 1978. - Heterosis. Beitrag 9 aus Genetik, Grundlagen, Ergebnisse und Probleme in Einzeldarstellungen. VEB Gustav Fischer Verlag, Jena.

Halliday R. B. 1978. - Genetic studies of meet ants (Iridomyrmex purpureus) Ph. D. thesis; University of Adelaide.

HARTL D., 1971. - Some aspects of natural selection in arrhenotokous populations. Am. Zool. 11, 309325.

HeLle W., 1965. - Inbreeding depression in an arrhenotokous mite Tetranychus urticae Koch. Ent. exp. appl. 8, 299-304.

Howell D. E., Usinger R. L., 1933. - Observations on the flight and length of the life of drone honeybees. Ann. Entomol. Soc. Am. 26, 239-246.

Kepeña L., 1963. - Beitrag zur Biologie der Drohnen des Stammes “ Tatranka ». 19. internat. Bienenz. Kongress Prag 1963, Teil II, 241-242.

1978. - Lebensdauer durch Inbreeding und Outbreeding unter Laborbedingungen erzielter Bienen. Genetik, Selektion und Reproduktion bei der Honigbiene. Apimondia-Verlag Bukarest, 56-59.

KERR W. E., 1969. - Some aspects of the evolution of social bees (Apidae). Evol. Biol. 3, 119-175. 
KHIscha W. D., 1978. - Einfluss von Inzucht und Zwischenlinienkreuzung auf Merkmale und Eigenschaften der Bienen. Genetik, Selektion und Reproduktion bei der Honigbiene. ApimondiaVerlag Bukarest, 60-68.

Kurennoi N. H., 1954. Flightactivity and sexual maturity of drones. Pchelovodstvo 12, 24-28.

Lester L. J., Selander R. K., 1978. - Population genetics of haplo-diploid insects. Gen. In press.

Maul V., 1969. - Die Ursache der Kreuzungsbarriere zwischen Apis mel. L. und Apis cerana Fab. 2. Eibefruchtung und Embryonalentwicklung. XII internat. Bienenz. Kongress, 515-516.

MAUTZ D., 1970. - Quantitative Untersuchungen über den Kommunikationseffekt der Schwänzeltänze bei Apis mellifica carnica. Dissertation, Johann Wolfgang Goethe Universität Frankfurt/Main 1970.

METCALF R. A. et al., 1975. - Low levels of genetic heterozygosity in Hymenoptera. Nature 257, 792-794.

MORTIZ, R.F.A., 1978. - Inzuchtdepression auf die Fitness der Drohnen (Apis mellifera capensis). Apidologie 9, 156-158.

OTT I., BRÜCKNER D., 1980. - Genetisch bedingte Unterschiede im Lernverhalten der Honigbiene (Apis mellifica carnica). Apidologie 11, 3-15.

Pamilo P. et al., 1978. - Low enzyme gene variability in Hymenoptera as a consequence of haplodiploidy. Hereditas 88, 93-99.

Ptrchner F., 1979. - Populationsgenetik in der Tierzucht. 2. Auflage, Verlag Paul Parey.

PLASS F., 1953. - Inzuchtwirkung und Heterosiseffekt bei der Honigbiene. AID 66, 49-68.

RoBERTS W. C., 1961. - Heterosis in the honeybee as shown by morphological characters in inbred and hybrid bees. Ann. Entomol. Soc. Am. 54, 878-882.

SNYDER T. P., 1974. - Lack of allozymic variability in three bee species. Evolution 28, 687-689.

TANTAWAY A. D., ReEvE E.C.R., 1956. - Studies in quantitative inheritance. IX. The effects of inbreeding at different rates in Drosophila melanogaster. Z. Indukt. Abst. Vererbungslehre 87, 648.

ToBari Y. N., MoRIWAKI D., 1963. - Maternal effects and heterosis in Drosophila ananassae. Gen. 48, 171-176.

WITHERELl P. C., 1970. - Behaviour of honeybees in glass-covered runways. Gleanings Bee Cult. 98, 664-668.

1971. - Duration of flight and interflight time of drone honeybees (Apis mellifera). Ann. Entomol. Soc. Am. 64, 609-612. 